\title{
A Performance Review of Recent Corner Detectors
}

\author{
Mohammad Awrangjeb and Guojun Lu \\ Gippsland School of Info. and Comm. Tech., Monash Univesrity, Churchill Vic 3842 Australia \\ E-mail: \{mohammad.awrangjeb, Guojun.Lu\}@monash.edu
}

\begin{abstract}
Contour-based corner detectors directly or indirectly estimate a significance measure (eg, curvature) on the points of a planar curve and select the curvature extrema points as corners. A number of promising contour-based corner detectors have recently been proposed. They mainly differ in how the curvature is estimated on each point of the given curve. As the curvature on a digital curve can only be approximated, it is important to estimate a curvature that remains stable against significant noises, for example, geometric transformations and compression, on the curve. Moreover, in many applications, for instance, in content-based image retrieval, a fast corner detector is a prerequisite. So, it is also a primary characteristic that how much time a corner detector takes for corner detection in a given image. In addition, different authors evaluated their detectors on different platforms using different evaluation systems. Evaluation systems that depend on human judgements and visual identification of corners are manual and too subjective. Application of a manual system on a large test database will be expensive. Therefore, it is important to evaluate the detectors on a common platform using an automatic evaluation system. This paper first reviews six most recent and highly performed corner detectors and analyse their theoretical running time. Then it uses an automatic evaluation system to analyse their performance. Both the robustness to noise and efficiency are estimated to rank the detectors.
\end{abstract}

\section{INTRODUCTION}

Contour-based corner detectors first perform edge detection, and then analyse the edges to find the locations of rapid changes in direction (ie, corners). They directly or indirectly estimate a significance measure (eg, curvature) on the points of a planar curve and select the curvature extrema points as corners. They have many applications in object extraction and image identification and retrieval. For example, Awrangjeb et al. [1] successfully applied corners for extraction of man-made objects such as buildings from aerial images.

The corner detectors mainly differ in how the curvature is estimated on each point of the given curve. As the curvature on a digital curve can only be approximated, it is important to estimate a curvature that remains steadfast against significant noises. Geometric transformations including rotation, scaling and cropping are often applied to images before they are posted on the Internet. Some geometric transformations, for instance, general affine transformations, not only change the size of the objects in the image, but also affect their shapes. For instance, a shear operation may change a rectangular shape to a parallelogram. Moreover, application of compression algorithms is a common operation to the image. All these operations may severely affect the extraction of planar curves from the image. Therefore, the extracted curves from an original image will be different from those which are extracted from one of its versions which may be geometrically transformed and compressed. As shown in Fig. 1, many of the extracted edges in the original 'Lena' image are either missed, cropped or extracted differently from the transformed and compressed images. For instance, look at the edges within the red ellipses. Although the compressed image looks almost the same as the original image, there is a significant difference between the extracted edges from the two images. Thus it is very important that a corner detector repeatedly extract the same corners in different versions of the same image. In the literature, this characteristic of a detector is termed as robustness [2].

A robust corner detector will be highly useful in various applications, for example, in content-based image retrieval and transformed image identification [3]. However, the running time of the detector is also important in such applications. The running time of different corner detectors primarily depends on how much time they take to estimate the curvature.

Recently, a number of promising contour-based detectors have been proposed [4], [2], [5], [6], [7], [8], [9]. They have outperformed one another and many other corner detectors. However, different authors used different evaluation systems. In fact, the traditional technique of performance evaluation involves human visual inspection procedure and does not estimate the robustness of a detector. For example, Mokhtarian and Mohanna [10] considered corners detected in the original images by a detector as the detected corners and corners identified by human subjects on the same original images as the ground truth. Then, the ground truth and detected corners were compared to evaluate the performance of that detector. Such a manual evaluation system evaluates accuracy of the detected corners with respect to human subjects, rather than robustness of the detector which indicates the ability of a detector to detect the same corners in different versions (geometric and signal processed) of the same image.

So, a manual evaluation system cannot be used for estimation of proper robustness of a detector due to following reasons. First, it is very hard to point out all corner locations in natural images by human intuition. Second, human eyes are unable to measure the corner strength. Third, the volume of work with a large image database for ground truth collection prohibits its adoption. Fourth, the human judgement may be biased. Finally, there is no standard procedure to collect the ground truth, eg, how many people should be involved and how to assess their decisions etc. Awrangjeb et al. [11] first proposed an automatic evaluation system which does not involve any human judgement and, therefore, can be applied on large image databases.

This paper first presents a brief review of six promising corner detectors followed by their theoretical complexity analysis. Then the detectors are evaluated using the automatic evaluation system [11] and ranked based on average repeatability, localization error and running time. 


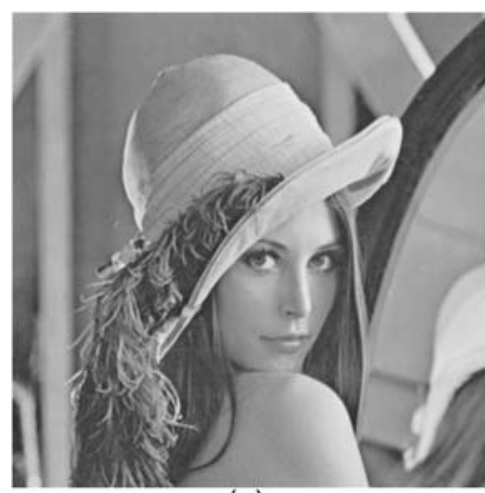

(a)

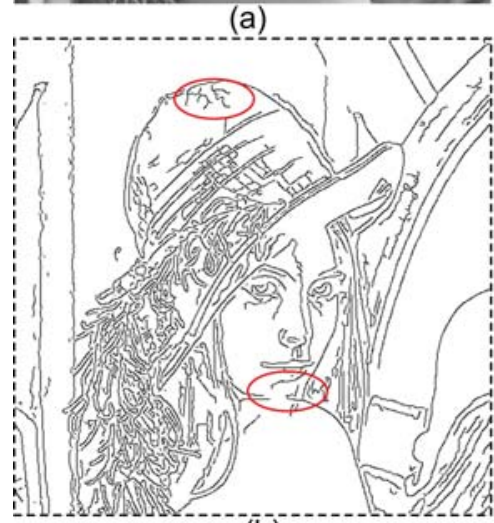

(b)

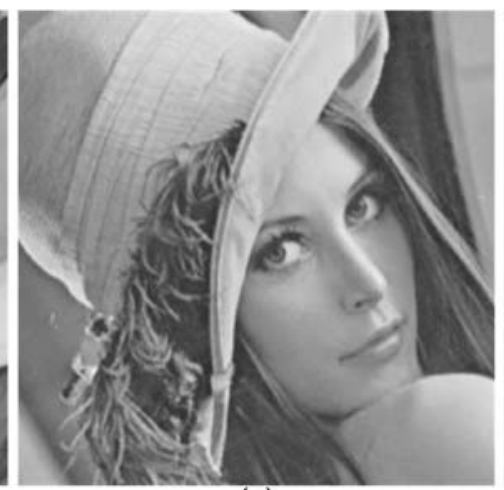

(c)
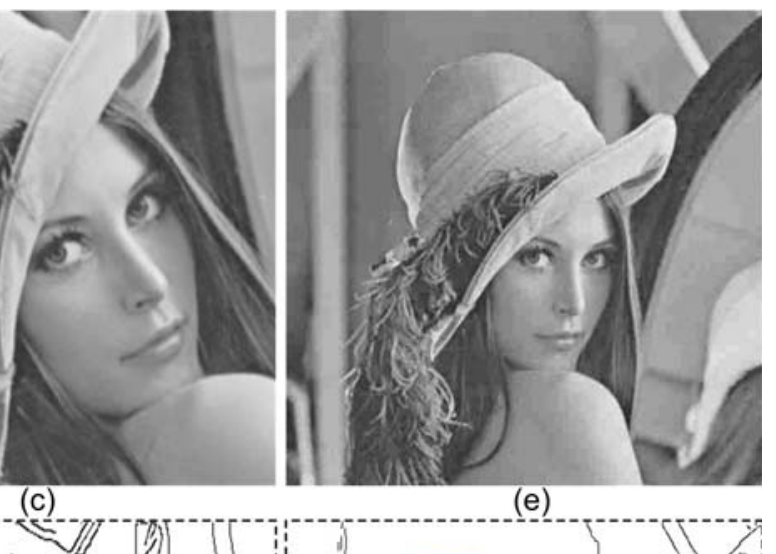

(e)

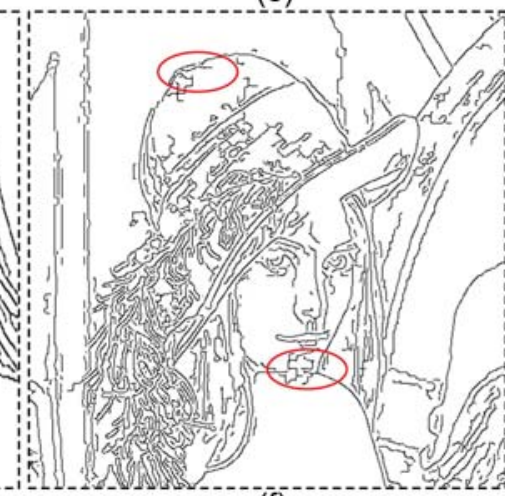

$(\mathrm{f})$

Fig. 1. Canny edges: (a) original 'Lena' image $\left(512 \times 512\right.$ pixels), (b) edges from (a), (c) transformed image $\left(421 \times 259\right.$ pixels: rotation $30^{\circ}$, scaling factors: 0.8 and 1.3 in $x$ and $y$ directions, respectively, and cropped), (d) edges from (c), (e) JPEG compressed image with a quality factor of 15 (512 $\times 512$ pixels), and (f) edges from (e). Here (c) and (d) are enlarged to show at the same size as others.

\section{CORNER Detectors}

A typical contour-based corner detector works as follows. First, in order to remove noise it uses the Gaussian convolution operation to smooth a curve $\Gamma(t)=(x(t), y(t))$, where $t$ is an arbitrary parameter, as follows.

$$
\Gamma(t, \sigma)=(x(t) \otimes G(t, \sigma), y(t) \otimes G(t, \sigma)),
$$

where $\Gamma(t, \sigma)$ is the smoothed curve, $\otimes$ is the convolution operation, $G(t, \sigma)$ is the Gaussian function with a smoothingscale $\sigma$. Let the smoothed curve be $\Gamma(t, \sigma)=\left(x_{s}(t), y_{s}(t)\right)$. Then the detector estimates a significance measure on each point of the smoothed curve. The local maxima points of the curvature function are the candidate corners. Finally, it may accept the candidate corners as final corners or apply some techniques to remove false and weak corners from the candidate corner set.

The corner detectors mainly differ in how the significance (also known as cornerness [8]) is estimated on each point of the given curve. In this section, the curvature estimation techniques by six recent corner detectors are discussed.

\section{A. Eigenvalue Detector}

Tsai et al. [4] used the smaller eigenvalue of the covariance matrix which is generated on each smoothed curve point $P_{i}$ over a region-of-support (RoS) $k$ as follows:

$$
\kappa(i)=\frac{1}{2}\left[c_{x x}+c_{y y}-\sqrt{\left(c_{x x}-c_{y y}\right)^{2}+4 c_{x y}^{2}}\right]
$$

where

$$
\begin{aligned}
c_{x x} & =\left[\frac{1}{2 k+1} \sum_{j=i-k}^{i+k} x_{s}(j)^{2}\right]-c_{x}^{2}, \\
c_{y y} & =\left[\frac{1}{2 k+1} \sum_{j=i-k}^{i+k} y_{s}(j)^{2}\right]-c_{y}^{2} \text { and } \\
c_{x y} & =\left[\frac{1}{2 k+1} \sum_{j=i-k}^{i+k} x_{s}(j) \cdot y_{s}(j)\right]-c_{x} \cdot c_{y},
\end{aligned}
$$

where $\left(c_{x}, c_{y}\right)$ is the geometrical centre of the smoothed curve segment $S_{i}=\left\{P_{i-k}, P_{i-k+1}, \ldots, P_{i+k}\right\}$ centred at $P_{i}$. They used a RoS size of $k=10$ on each side of $P_{i}$.

\section{B. DoG (Difference of Gaussian) Detector}

Zhang et al. [6] used the intuitive idea that a curve is more smoothed when a higher $\sigma$ value is used. So, they smoothed the original curve using two $\sigma$ values (for example, $\sigma_{1}=2$ and $\sigma_{2}=1.5 \sigma_{1}$ ) and obtained the difference between the two smoothed curves as cornerness. Using the distributive law of convolution, this operation is equivalent to smoothing the original curve by the difference of two Gaussian functions. Following (1)

$$
\Gamma\left(t, \sigma_{d}\right)=\left(x(t) \otimes G\left(t, \sigma_{d}\right), y(t) \otimes G\left(t, \sigma_{d}\right)\right),
$$

where $G\left(t, \sigma_{d}\right)=G\left(t, \sigma_{2}\right)-G\left(t, \sigma_{1}\right)$ is the Difference of Gaussian (DoG) function. Then they estimated the curvature using the norm

$$
\kappa(i)=x_{s}(i)^{2}+x_{s}(i)^{2},
$$

where $\left(x_{s}(i), y_{s}(i)\right)$ is the smoothed curve using (4). 


\section{CPDA (Chord-to-Point Distance Accumulation) and Fast- CPDA Detectors}

Awrangjeb and $\mathrm{Lu}$ [5] used the chord-to-point distance accumulation (CPDA) technique to estimate a significance measure $h_{L}(i)$ at $P_{i}$ using a chord of length $k$. The chord moves on each side of $P_{i}$ at most $k$ points.

$$
h_{L}(i)=\sum_{j=i-k+1}^{i-1} d_{i, j},
$$

where $d_{i, j}$ is the perpendicular distance from $P_{i}$ to the chord. The CPDA detector used 3 chords of lengths $k_{1}=10$, $k_{2}=20$ and $k_{3}=30$ and estimated 3 corresponding curvature functions $h_{1}(i), h_{2}(i)$ and $h_{3}(i)$. Then it normalised each individual curvature function and multiply them together

$$
\kappa(i)=h_{1}^{\prime}(i) \cdot h_{2}^{\prime}(i) \cdot h_{3}^{\prime}(i), \text { where } h_{l}^{\prime}(i)=\frac{h_{l}(i)}{\max \left(a b s\left(h_{l}(i)\right)\right)},
$$

where $1 \leq l \leq 3$.

Since the distance accumulation using (6) on all the curvepoints required a significant time, Awrangjeb and $\mathrm{Lu}$ [7] later used a candidate point set $S_{c}$ by applying the DoG function discussed above $\left(\sigma_{1}=3, \sigma_{2}=4\right)$. Then the CPDA curvature was estimated only on $S_{c}$. The improved detector is known as the fast-CPDA detector.

\section{GCM (Gradient Correlation Matrices) Detector}

Zhang et al [8] used the determinants of the gradient correlation matrices $(\mathrm{GCM})$ :

$$
\kappa(i)=\sum_{j=i-k+1}^{i-1}\left|\begin{array}{cc}
\dot{x}_{s}(j)^{2} & \dot{x}_{s}(j) \dot{y}_{s}(j) \\
\dot{x}_{s}(j) \dot{y}_{s}(j) & \dot{y}_{s}(j) \dot{y}_{s}(j)
\end{array}\right|,
$$

where $\dot{x}_{s}(j)$ and $\dot{y}_{s}(j)$ are first order derivatives with respect to $j$. They used $k=1$.

\section{E. CTAR (Chord to Triangular Arms Ratio) Detector}

Sadat et al. [9] recently applied a simple triangular theory for curvature estimation. Let the two end points of the RoS for $P_{i}$ be $P_{i-k}$ and $P_{i+k}$. The curvature at $P_{i}$ is computed using the three arm distances of the triangle $P_{i} P_{i-k} P_{i+k}$ as

$$
\kappa(i)=\frac{d_{1}}{d_{2}+d_{3}},
$$

where $d_{1}=\left|P_{i-k} \cdot P_{i+k}\right|, d_{2}=\left|P_{i} \cdot P_{i-k}\right|$ and $d_{2}=\left|P_{i} \cdot P_{i+k}\right|$ are the simple Euclidean distances. The curvature value at $P_{i}$ decreases if the corner at $P_{i}$ is sharp and increases if the corner at $P_{i}$ is round. Sadat et al. [9] used $k=3$. They coined the detector as CTAR (chord to triangular arms ratio).

\section{Computational COMPleXity}

For computational complexity, only the multiplication, division, square and square root operations are considered. Each of the detectors takes $m n$ operations for smoothing the curve and $n$ operations to obtain the curvature extrema points, where $m$ is the width of the Gaussian smoothing function and $n$ is the number of curve-points. The estimation of curvature takes different number of operations as discussed below.
TABLE I. COMPUTATIONAL COSTS IN NUMBER OF TOTAL OPERATIONS. SYMBOLS: $m$ IS THE WIDTH OF THE GAUSSIAN SMOOTHING FUNCTION, $n$ IS THE NUMBER OF CURVE-POINTS AND $n_{c}$ IS THE NUMBER OF CANDIDATE CURVE-POINTS $\left(n_{c} \ll n\right)$.

\begin{tabular}{l|l}
\hline \hline Detectors & Operations \\
\hline Eigenvalue [4] & $m n+16 n$ \\
\hline DoG [6] & $m n+3 n$ \\
\hline CPDA [5] & $m n+408 n$ \\
\hline Fast-CPDA [7] & $m n+2 n+408 n_{c}$ \\
\hline GCM [8] & $m n+8 n$ \\
\hline CTAR [9] & $m n+10 n$ \\
\hline \hline
\end{tabular}

For the Eigenvalue detector [4], the computations of $x_{j}^{2}$, $y_{j}^{2}$ and $x_{j} . y_{j}$ in (3) cost a total of $3 n$ operations. For each point $P_{i}$, the computation of $\left(c_{x}, c_{y}\right)$ needs 2 divisions and there are additional 10 operations in (2) to (3). Consequently, the total number of operations for curvature estimation using (2) is $15 n$.

The smoothing operation by the DoG detector [6] using (4) needs the similar number of operations as the other detectors need using (1). So, it needs only $2 n$ operations to calculate its curvature using (5), which has made it one of the fastest corner detectors in the literature.

By the CPDA detector [5], a total of 7 operations is required for each distance computation using (6) and there are $k-1$ such distances for each chord. The CPDA detector uses 3 chords and on average there are 20 chord movements (ie, $k=20$ ) for $P_{i}$. So there is a total of $21 n(k-1)=399 n$ computations in (6). For each chord, there are $2 n$ computations for normalization and an additional $2 n$ operations for computing the curvature product using (7). As a result, the total computations by the CPDA detector to estimate the curvature function is $407 n$.

The fast-CPDA detector [7] needs $2 \mathrm{n}$ operations to find the candidate point set using (5). Let $n_{c}$ be the number of the candidate points. Therefore, it needs a total of $2 n+407 n_{c}$ operations to estimate curvature. Since $n_{c} \ll n$, the fast-CPDA detector takes significantly lower time than the CPDA detector.

For the GCM detector [8], the computation of $x$ and $y$ derivatives takes a total of $2 n$ operations. The computation of the 3 entries in the matrix of (8) takes another $3 n$ operations. Each determinant operation requires 2 multiplications. So the total cost to calculate the GCM significance is $7 n$.

For the CTAR detector [9], each Euclidean distance computation in (9) needs 3 operations. So there are $9 n$ operations altogether to estimate the curvature function for a curve. Such a low computational cost has made the CTAR detector as one of the fastest contour-based detectors.

Table I shows the computational costs of all six promising detectors. For each detector, Table I also includes $m n$ operations for smoothing the curve and $n$ operations to obtain the curvature extrema points.

\section{Performance Study}

In this section, the performance of the six promising detectors are empirically tested. The automatic performance evaluation system introduced in [11] is followed. In the automatic system, corners detected in the original images by 


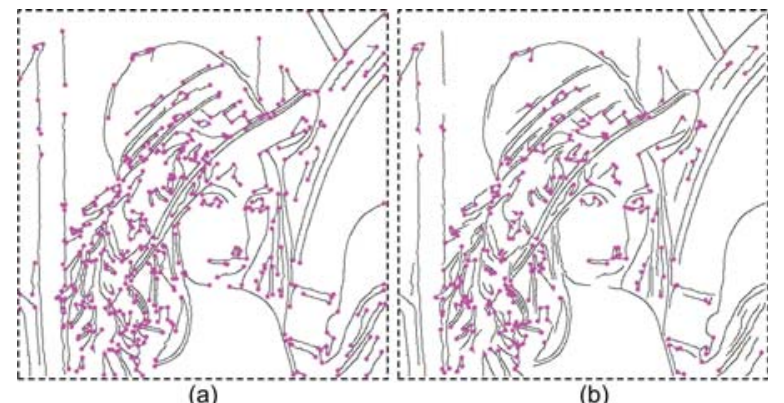

(a)
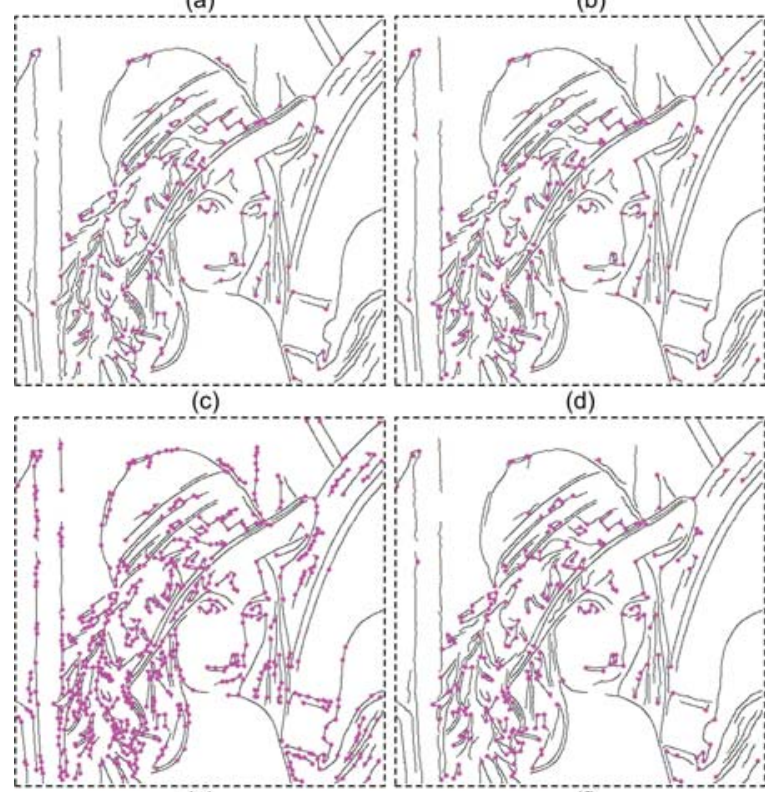

(e)

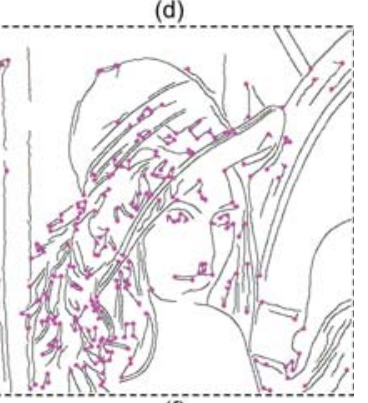

(f)

Fig. 2. Corners on the original 'Lena' image: detected by (a) Eigenvalue [4], (b) DoG [6] (c) CPDA [5], (d) Fast-CPDA [7], (e) GCM [8] and (f) CTAR [9] detectors. Eigenvalue and GCM detectors detected a large number of false and weak corners. Only long Canny edges are shown.

a detector are considered as reference corners and corners detected by the same detector in the test images, which were signal processed and geometrically transformed, are taken as test corners. The reference and test corners are then compared to evaluate the robustness of that detector. During comparisons the reference corners are transformed using the known transformations (between the original and test images) in order to find their correspondences with the test corners. A maximum localization error of $L_{\max }$ pixels is allowed while finding the correspondences.

Three evaluation metrics: average repeatability $R_{\text {avg }}$, localization error $L_{e}$ and running time are used for robustness and efficiency evaluations. Repeatability $R$ indicates how stable the detected corners are under different geometric transformations and signal processing operations. Average repeatability is defined as:

$$
R_{a v g}=\frac{N_{r}}{2}\left(\frac{1}{N_{0}}+\frac{1}{N_{t}}\right) .
$$

where $N_{0}$ and $N_{t}$ are the number of corners in the original and test images, respectively and $N_{r}$ is the number of repeated corners between them. Localization error shows how accurately a detected corner is localized by the detector, and it
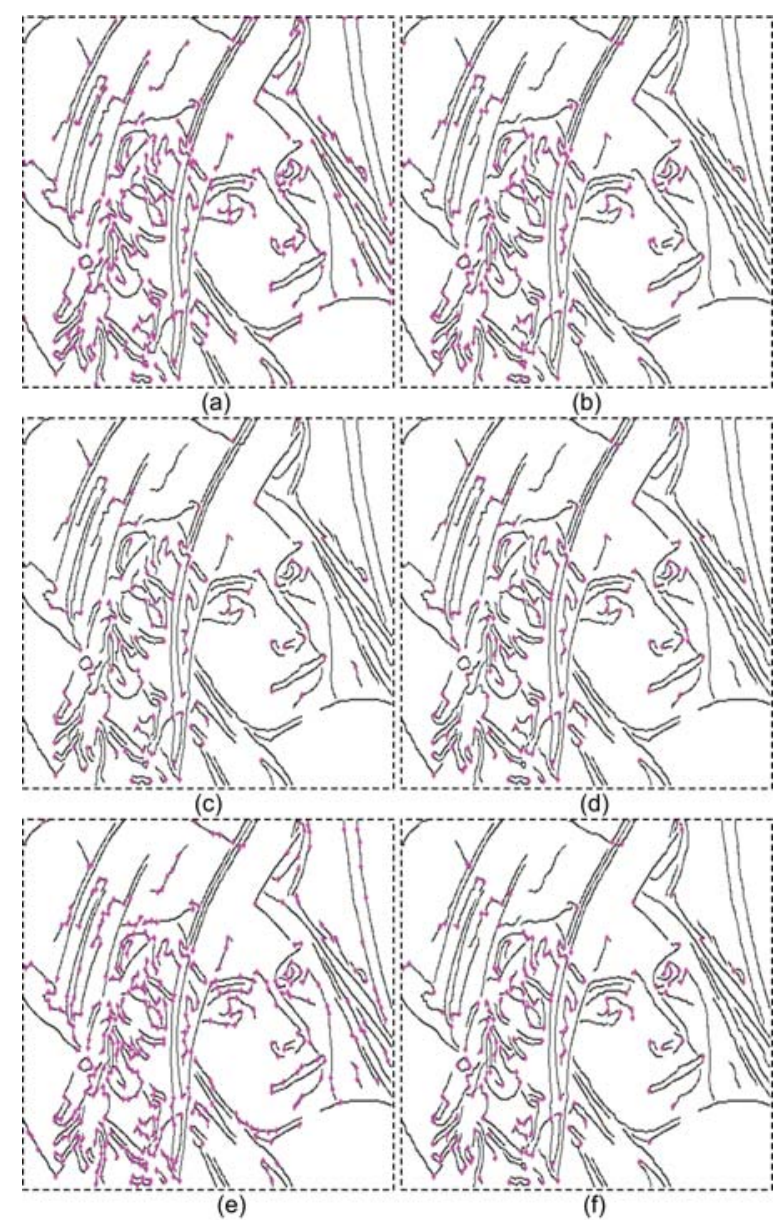

(e)

Fig. 3. Corners on the transformed 'Lena' image, shown in Fig. 1(b): detected by (a) Eigenvalue [4], (b) DoG [6] (c) CPDA [5], (d) Fast-CPDA [7], (e) GCM [8] and (f) CTAR [9] detectors. Eigenvalue and GCM detectors detected a large number of false and weak corners. Only long Canny edges are shown.

is measured using the root-mean-square-error (RMSE) of the detected corners:

$$
L_{e}=\sqrt{\frac{1}{N_{r}} \sum_{i=1}^{N_{r}}\left[\left(x_{t i}-x_{o i}\right)^{2}+\left(y_{t i}-y_{o i}\right)^{2}\right]} .
$$

Here $\left(x_{o i}, y_{o i}\right)$ and $\left(x_{t i}, y_{t i}\right)$ are the original and test positions of the $i$-th repeated corner.

The data set had a total of 23 different original $512 \times 512$ grey-scale images including some artificial images like 'Block' and real world images like 'Lena', 'Leaf', 'House' and 'Lab'. All of the above original images were collected from standard data sets [12], [13]. The data set employed had a total of 8694 affine transformed and signal processed (test) images, which were obtained by applying the following seven approaches of operations on each original image:

$\diamond$ rotation at 18 different angles $\theta$ in $\left[-90^{\circ},+90^{\circ}\right]$ at $10^{\circ}$ apart, excluding $0^{\circ}$;

$\diamond \quad$ uniform (U) scaling factors $s_{x}=s_{y}$ in $[0.5,2.0]$ at 0.1 apart, excluding 1.0;

$\diamond \quad$ non-uniform (NU) scaling factors $s_{x}$ in $[0.7,1.3]$ and 


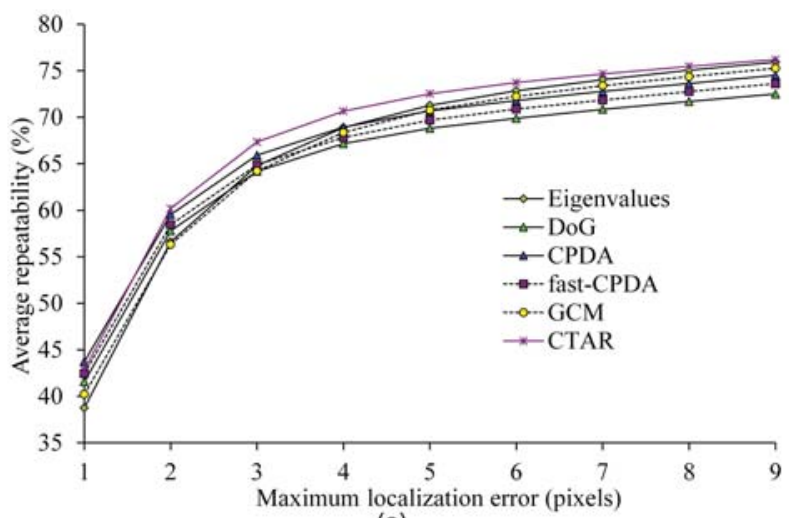

(a)

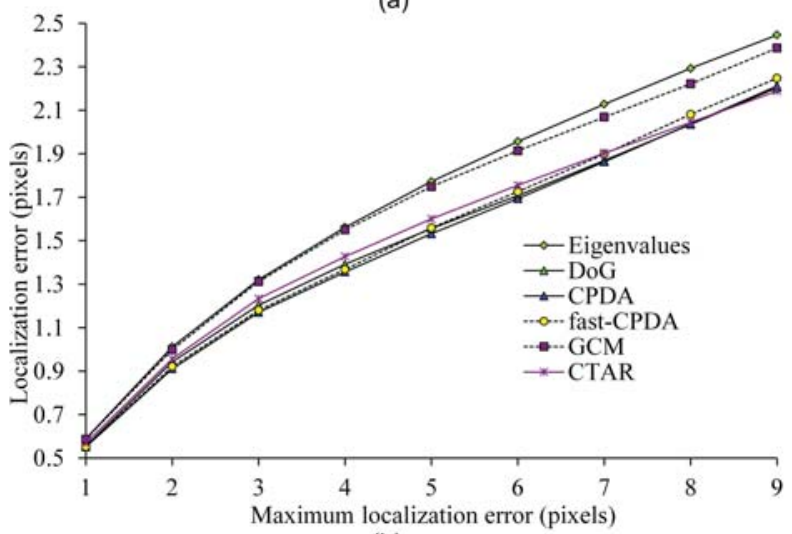

(b)

Fig. 4. Robustness (average repeatability and localization error) of detectors at different maximum localization error.

$s_{y}$ in $[0.5,1.8]$, at 0.1 apart, excluding the cases when $s_{x}=s_{y}$;

$\diamond$ combined transformations (rot.-scale): $\theta$ in $\left[-30^{\circ},+30^{\circ}\right]$ at $10^{\circ}$ apart, excluding $0^{\circ}$, followed by uniform or non-uniform scaling factors $s_{x}$ and $s_{y}$ in $[0.8,1.2]$ at 0.1 apart;

$\diamond$ JPEG lossy compression at 20 quality factors in $[5,100]$, at 5 apart;

$\diamond \quad$ zero mean white Gaussian noise at 10 variances in $[0.005,0.05]$ at 0.005 apart; and

$\diamond \quad$ shearing factors $s_{h x}$ and $s_{h y}$ in $[0,0.012]$ at 0.002 apart, excluding the one when $s_{h x}=s_{h y}=0.0$.

Therefore, the data set had a total of 414 rotated, 345 uniform-scaled, 2691 non-uniform scaled, 3450 rotated and scaled transformed images. It also had 460 JPEG compressed, 230 Gaussian noise-induced and 1104 sheared images. Note that transformations comprising rotations were also followed by cropping that removed the outer black parts. Consequently, many detected corners in the original images were cropped off in the test images for the transformations involving rotations.

For obtaining running time, each detector was applied on all original images $(512 \times 512)$ and then the average time was estimated. The machine was an Intel Xeon CPU 3.30GHz, 16GB RAM, Windows 7, MATLAB 2012a.

Fig. 2 shows some corner detection examples on the 'Lena' image by different detectors. Fig. 3 shows the detected corners on the transformed image shown in Fig. 1(c). As can be seen in both figures, the Eigenvalue and GCM detectors detected a large number of false corners. All other detectors detected almost all the prominent corners.

Most of the applications (for example, for identification of appropriate image patches in feature-point based image copyright protection schemes [14]) require high repeatability and low localization error of the detected corners. There are also other applications (for example, extraction of building roof lines [1]) that require high repeatability, but may allow some localization error. Therefore, the robustness of the detectors were estimated when the maximum localization error $L_{\max }$ was varied. Fig. 4 shows the average repeatability and localization error under different $L_{\max }$ values. The results are averaged over the whole database. It is observed that when $L_{\max }$ is varied from 1 to 3 pixels, $R_{a v g}$ increases dramatically for all detectors from around $40 \%$ to around $65 \%$. Thereafter, $R_{\text {avg }}$ increases gradually. In contrast, $L_{e}$ increased moderately at all $L_{\max }$ values.

For example, Fig. 5 shows the matched corners, from the CTAR detector [9], between the original (258 corners) and the transformed (177 corners) 'Lena' images. Many corners detected in the original image were either missed or cropped off in the transformed image. At $L_{\max }=3$ pixels, there were 65 matched corners (cyan lines) with $L_{e}=1.42$ pixels, when the average error per matched corner is $\frac{1.42}{65}=0.022$ pixels. However, at $L_{\max }=5$ pixels, there were 10 additional matched corners (yellow lines) with $L_{e}=1.89$ pixels; which is an increase of $\frac{1.89-1.42}{10}-0.022=0.025$ pixels per extra matched corner.

As can be seen in Fig. 4, at $L_{\max }=3$ pixels, the localization error for all detectors is around 1.2 pixels (average over all images in the database). Therefore, the optimal value of $L_{\max }$ can be considered to be at 3 pixels when high repeatability and low localization error can be observed.

Consequently, for applications which require high corner detection performance, the CTAR, CPDA and fast-CPDA detectors are good choices. In contrast, for applications which require high $R_{a v g}$ values, but may allow some localization errors, any of the six detectors can be used.

Overall, in terms of repeatability the CTAR detector performed the best and the DoG detector performed the worst among the six detectors. In terms of localization, the CPDA detector performed the best and the Eigenvalue detector showed the worst performance. The Eigenvalue and GCM detectors have higher localization errors than the other detectors. The Eigenvalue detector uses the covariance matrices and the GCM detector used the first order derivatives of curve-point locations. Since the covariance matrices and derivatives are sensitive to noises in curves, these detectors offer high $L_{e}$ and detect many spurious corners (see Fig. 2).

Table II shows the ranking of the six detectors based on average repeatability and localization error at $L_{\max }=3$ pixels and execution time per image. In terms of robustness, the CTAR, CPDA and fast-CPDA detectors are better than the other detectors. In terms of running time, the CTAR, GCM and fast-CPDA detectors are faster. Note that the execution time shown in Table II does not match with the 


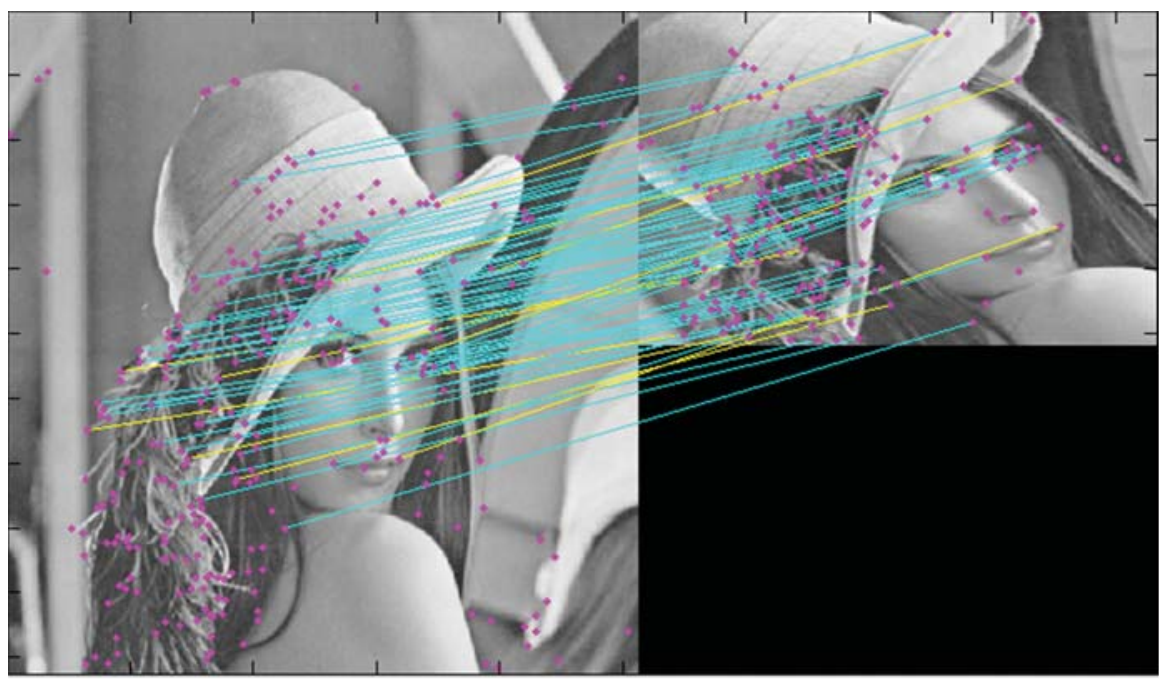

Fig. 5. Corners matched by the automatic matching algorithm [11]. Corners were detected by the CTAR detector [9] on the original 'Lena' image (left) and its transformed version (right) shown in Fig. 1(c). The 65 matching corners at $L_{\max }=3$ pixels are connected by the cyan coloured lines. The additional 10 matching corners found at $L_{\max }=5$ pixels are connected by the yellow coloured lines.

TABLE II. RANKING OF THE DETECTORS BASED ON AVERAGE REPEATABILITY, LOCALIZATION ERROR AND RUNNING TIME (PER IMAGE).

\begin{tabular}{c|l|l|l}
\hline \hline Ranks & Repeatability (\%) & Localization error (pixels) & Execution time (seconds) \\
\hline 1 & CTAR [9] (67.34) & CPDA [5] (1.17) & CTAR [9] (0.00232) \\
\hline 2 & CPDA [5] (65.91) & Fast-CPDA [7] (1.18) & GCM [8] (0.00428) \\
\hline 3 & Fast-CPDA [7] (64.89) & DoG [6] (1.20) & Fast-CPDA [7] $(0.00703)$ \\
\hline 4 & Eigenvalue [4] (64.79) & CTAR [9] (1.23) & DoG [6] $(0.00718)$ \\
\hline 5 & GCM [8] (64.21) & GCM [8] (1.31) & CPDA [5] $(0.01677)$ \\
\hline 6 & DoG [6] (64.19) & Eigenvalue [4] (1.32) & Eigenvalue [4] $(0.07492)$ \\
\hline \hline
\end{tabular}

theoretical computational costs shown in Table I, especially the Eigenvalue detector became the slowest detector, since the estimation of the eigenvalues of covariance metrics using a $10 \times 10$ neighbourhood around each pixel took a significant time. The discrepancy was partly due to different ways of implementations of the detectors by the respective authors.

\section{Conclusions}

In this paper, a performance analysis of six promising contour-based detectors has been presented. Experientially, it has been observed that while the CTAR, CPDA and fast-CPDA detectors are more robust, the CTAR, GCM and fast-CPDA detectors are faster. This study would give an idea as to which detector best suits a particular application. In applications which require robust and fast corner detection techniques, the CTAR, CPDA and fast-CPDA detectors would obviously be the first choices. In other applications, any of the six detectors may be employed.

\section{REFERENCES}

[1] M. Awrangjeb, C. Zhang, and C. S. Fraser, "Building detection in complex scenes thorough effective separation of buildings from trees,' Photogrammetric Engineering \& Remote Sensing, vol. 78, no. 7, pp. 729-745, 2012

[2] M. Awrangjeb and G. Lu, "An improved curvature scale-space corner detector and a robust corner matching technique for transformed image identification," IEEE Trans. Image Process., vol. 17, no. 12, pp. 24252441, Dec 2008

[3] _ , "Techniques for efficient and effective transformed image identification," Journal of Visual Communication and Image Representation, vol. 20 , no. 8 , pp. 511-520, 2009.
[4] D.-M. Tsai, H. T. Hou, and H. J. Su, "Boundary-based corner detection using eigenvalues of covariance matrices," Pattern Recognition Letters, vol. 20, no. 1, pp. 31-40, Jan 1999.

[5] M. Awrangjeb and G. Lu, "Robust image corner detection based on the chord-to-point distance accumulation technique," IEEE Trans. Multimedia, vol. 10, no. 6, pp. 1059-1072, Oct 2008.

[6] X. Zhang, H. Wang, M. Hong, L. Xu, D. Yang, and B. C. Lovell, "Robust image corner detection based on scale evolution difference of planar curves," Pattern Recognition Letters, vol. 30, no. 1, pp. 449-455, Mar 2009.

[7] M. Awrangjeb, G. Lu, C. S. Fraser, and M. Ravanbakhsh, "A fast corner detector based on the chord-to-point distance accumulation technique," in Proc. Digital Image Computing: Techniques and Applications, Melbourne, Australia, Dec 2009, pp. 519-525.

[8] X. Zhang, H. Wang, A. W. B. Smith, X. Ling, B. C. Lovell, and D. Yang, "Corner detection based on gradient correlation matrices of planar curves," Pattern Recognition, vol. 43, no. 4, pp. 1207-1223, Apr 2010.

[9] R. M. N. Sadat, S. W. Teng, and G. Lu, "An effective and efficient contour-based corner detector using simple triangular theory," in Proc. 19th Pacific Conference on Computer Graphics and Applications, Goslar, Germany, 2011, pp. 37-42.

[10] F. Mokhtarian and F. Mohanna, "Performance evaluation of corner detectors using consistency and accuracy measures," Computer Vision and Image Understanding, vol. 102, no. 1, pp. 81-94, Apr. 2006.

[11] M. Awrangjeb, G. Lu, and M. Murshed, "An affine resilient curvature scale-space corner detector," in Proc. IEEE International Conference on Acoustics, Speech, and Signal Processing, vol. 1, Hawaii, USA, Apr. 2007, pp. 1233-1236.

[12] F. A. P. Petitcolas. Photo database, http://www.petitcolas.net/fabien/watermarking/image_database/index.html.

[13] USC-SIPI. The usc-sipi image database, http://sipi.usc.edu/database/.

[14] M. Awrangjeb and G. Lu, "A robust content-based watermarking technique," in Proc. IEEE International Workshop on Multimedia Signal Processing, Cairns, Australia, Oct. 2008, pp. 713-718. 